INPLASY

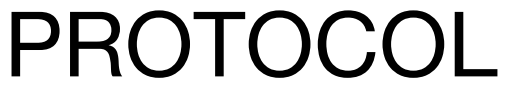

To cite: Zhang et al. The efficacy of mTORi based immunosuppression In Liver

Transplantation: A Systematic

Review and Meta-analysis.

Inplasy protocol 202210058.

doi:

10.37766/inplasy2022.1.0058

Received: 12 January 2022

Published: 12 January 2022

Corresponding author:

Zhang Gongming

1139688021@qq.com

Author Affiliation:

Capital Medical University,

Beijing Youan Hospital.

Support: Beijing You 'an

Hospital.

Review Stage at time of this submission: Data analysis -

Completed but not published.

Conflicts of interest:

None declared.

\section{The efficacy of mTORi based immunosuppression In Liver Transplantation: A Systematic Review and Meta-analysis}

Zhang, G1; Duan, B2; Li, G³.

Review question / Objective: Sirolimus (SRL) and everolimus (EVR) are a part of the mammalian target of rapamycin inhibitors (mTORi), a novel immunosuppression protocol that can reduce recurrence rate following liver transplantation (LT) for hepatocellular carcinoma (HCC). However, their efficacy and safety have not been fully explored. Herein, we performed a systematic review and meta-analysis to investigate the side effect of mTORi and CNI in HCC patients after LT.

Information sources: A comprehensive search was performed using MEDLINE, EMBASE, and Cochrane Library databases up to October 2021.

INPLASY registration number: This protocol was registered with the International Platform of Registered Systematic Review and Meta-Analysis Protocols (INPLASY) on 12 January 2022 and was last updated on 12 January 2022 (registration number INPLASY202210058).

\section{INTRODUCTION}

Review question / Objective: Sirolimus (SRL) and everolimus (EVR) are a part of the mammalian target of rapamycin inhibitors (mTORi), a novel immunosuppression protocol that can reduce recurrence rate following liver transplantation (LT) for hepatocellular carcinoma (HCC). However, their efficacy and safety have not been fully explored. Herein, we performed a systematic review and meta-analysis to investigate the side effect of mTORi and CNI in HCC patients after LT.

Condition being studied: Liver cancer is the fourth most common cause of cancer- 
related death and sixth in terms of incident cases worldwide. For patients who are not eligible for surgery, liver transplantation (LT) is usually the best choice for treatment. Although LT has many advantages compared with other treatments, it still has some limitations, such as organ rejection caused by the donor and perioperative complications. Moreover, it is a technically demanding procedure. Also, patients who undergo LT are required to take immunosuppressants after surgery. Tacrolimus and cyclosporine $A$ in calcineurin inhibitors (CNI) are the most widely used immunosuppressors for LT recipients. However, their efficacy and drug safety have not been fully explored. Previous studies have shown that CNI are always accompanied by short-term or long-term adverse effects, some of which may include tumor recurrence. Consequently, searching for a new drug with equal or better efficacy but fewer adverse reactions for immunosuppression is needed. mTORi is a novel immunosuppressant that has a different mechanism compared to normal immunosuppressive drugs. The two main mTORi regimens, sirolimus and everolimus, have been found to have a low influence on renal function. In addition, they can improve the survival rate and reduce the recurrence of tumors in LT patients for HCC. Besides, it has also been suggested that mTORi are associated with a low incidence of AEs and rejection. Herein, we performed a systematic review and metaanalysis to investigate the side effect of mTORi and CNI in HCC patients after LT. Specifically, we reviewed the potential efficacy and safety of mTORi in HCC transplant patients and its impact on HCC recurrence and overall mortality.

\section{METHODS}

Search strategy: A comprehensive search was performed using MEDLINE, EMBASE, and Cochrane Library databases up to October 2021. The following subject terms and free terms were used:'sirolimus', 'everolimus','mammalian target of rapamycin inhibitors', 'mTOR inhibitors', 'hepatic transplantation' AND 'liver transplantation', 'Liver Grafting', 'Liver Neoplasms', 'Hepatocellular Carcinomas'. There were no restrictions on publication date and language.

Participant or population: Patients with liver cancer after LT. 11280

Intervention: Two different kinds of immunosuppression including mTORi and $\mathrm{CNI}$ after liver transplantation.

Comparator: Two groups' different outcomes were compared. The primary outcome of interest was recurrence rate and overall mortality. Secondary endpoints were acute rejection and AEs. Acute rejection (including biopsy proved acute rejection, BPAR), HAT, graft loss, microvascular invasion/vascular invasion, AEs, acne, anemia, Abnormal healing, biliary complications, cardiovascular disease, dyslipidemia, depression, diabetes, dysmenorrhea, diarrhea, edema, erythema, fatigue, fever, gastrointestinal symptoms, headache/migraine, hy perkalemia, hypertension, hyperglycemia, hypercholesterolemia, incisional hernia, infection, leukopenia, malignancies, mouth ulceration/stomatitis/ oral sores, nausea, nasopharyngitis, pain, PVT, pyrexia, proteinuria, pneumonitis, pleural effusion, pruritis, rash, renal failure, sepsis, thrombocytopenia, and vascular invasion.

Study designs to be included: A total of 4930 studies were retrieved from PubMed, Embase, and the Cochrane Library. EndNote software was then applied to exclude the duplicated studies. The rest of the studies were screened based on the title/abstract. A total of 1080 full-text articles were identified and assessed for eligibility. Finally, according to inclusion and exclusion criteria, 41 articles with 11280 HCC patients were included in our meta-analysis.

Eligibility criteria: Inclusion criteria were: Comparative studies based on mTORi and $\mathrm{CNI}$ as immunotherapy regimens used in patients with liver cancer after LT. 
Information sources: A comprehensive search was performed using MEDLINE, EMBASE, and Cochrane Library databases up to October 2021.

Main outcome(s): Twenty-three studies (6751 subjects) reported on recurrence rate. Meta-analysis demonstrated a significant improvement with mTORi therapy at recurrence rate (RR:0.76, 95\% CI:0.66-0.87). Figure 3 shows the corresponding forest plots and $Q, 12$ and $\tau 2$ statistics. There was moderate heterogeneity in recurrence rate $(12=48 \%)$.

Quality assessment / Risk of bias analysis: As different types of research literature have different quality assessment criteria, the observational studies were assessed by two independent reviewers (Zhang and Duan) using the Cochrane Risk of Bias Tool. An observational study may have a lower quality compared with a randomized controlled trial (RCT). In case of disagreements, a third author (LGM) was invited.

Strategy of data synthesis: None.

Subgroup analysis: None.

Sensitivity analysis: None.

Country(ies) involved: China.

Keywords: Sirolimus; everolimus; mTORi; liver transplantation; hepatocellular carcinomaliver transplantation; CNI; mTORi; immunosuppression.

Contributions of each author:

Author 1 - Zhang Gongming drafted the manuscript.

Author 2 - Duan Binwei analysed the data.

Author 3 - Li Guangming designed the study. 